\title{
Infants can rapidly form new categorical representations
}

\author{
REBECCA J. RIBAR \\ University of Wisconsin, Milwaukee, Wisconsin \\ LISA M. OAKES \\ University of Iowa, Iowa City, Iowa \\ and \\ THOMAS L. SPALDING \\ University of Alberta, Edmonton, Alberta, Canada
}

\begin{abstract}
Young infants learn common categorical distinctions, such as animals versus vehicles. But can they, like adults, rapidly form new categories, such as black-and-white animals? To answer this question, 6-, 10-, and 13-month-old infants were familiarized with four land animals that were black and white in coloring (e.g., a zebra and a black-and-white tiger) and then were tested with novel animals and a truck. The infants responded to an exclusive category that apparently included only black-and-white animals, suggesting that they formed a new categorical representation during familiarization. A comparison group of infants familiarized with a set of land animals that were more variable in coloring (e.g., a pale yellow horse and a yellow-and-brown tiger) formed a very general categorical representation that included many different kinds of animals, regardless of coloring. Therefore, like adults, infants rapidly form new categorical representations in response to the context.
\end{abstract}

Recently, there has been increasing interest in the origins and early development of categorization. This interest stems from the recognition that infants need a means for organizing and making sense of the enormous number of objects they encounter and reflects an interest in whether category formation is based on similar processes throughout the lifespan (e.g., Oakes \& Madole, 2000; Quinn \& Eimas, 1997). Many studies have shown that infants can respond to adult-defined categories, such as animals versus vehicles (Mandler \& McDonough, 1993), dogs versus cats (Quinn, Eimas, \& Rosenkranz, 1993), and food versus furniture (Ross, 1980), demonstrating that we have sensitive experimental measures to tap those abilities. Although such results provide a critically important foundation for our understanding of the early development of categorization, they tell us little about the processes of categorization or the information infants use to form category representations.

A portion of these data was presented at the meeting of the Cognitive Development Society, October 1999, Chapel Hill, NC. This research and preparation of this manuscript was made possible by a Central Investment Fund for Research Enhancement grant by the University of Iowa and by NICHD Grant HD36060 awarded to L.M.O. We thank Steve Luck, Larissa Samuelson, and John Spencer for extremely helpful comments on drafts of this article and Kathleen Kannass, Cathlin McCollough, and the undergraduates at the University of Iowa Infant Perception and Cognition lab for their help with the data collection and coding phases of this project. Correspondence should be addressed to L. M. Oakes, Department of Psychology, University of Iowa, Iowa City, IA 52242 (email: lisa-oakes@uiowa.edu).
Research with adults, in contrast, focuses on just these questions. In a typical categorization study, adults are presented with a set of stimuli (often, novel stimuli that conform to an unfamiliar categorical distinction) under different conditions, and the categorical relations formed in those conditions are observed. These studies have revealed that adults' categorization is highly context dependentfor example, adults judge different items as typical category members in different contexts (Roth \& Shoben, 1983), they categorize objects more easily if the features of those objects are consistent with their background knowledge (Spalding \& Murphy, 1996), and they perceive such properties as object color differently as a function of how items are categorized (Goldstone, 1995). Thus, the adult literature has focused on categorization as a flexible process responsive to the context.

In the present investigation, we asked whether infants can form novel categorical representations of realistic items that may be included in adult-defined categories (in this case, land animals ${ }^{1}$ ). Previous research investigating infants' attention to such categories (e.g., dogs or vehicles) cannot establish whether infants respond on the basis of categorical representations that they possessed before coming into the lab or on the basis of categorical representations that they formed on line. For example, infants familiarized with a set of cats may form a categorical representation of cat at that moment, or they may recall a previously formed categorical representation of cat. Therefore, even when infants' responding seems to reflect an underlying stable concept, they may actually have formed 
a novel categorical representation during the experimental session. Indeed, studies have revealed that infants' categorization of objects from familiar categories (such as $\operatorname{dog} s$ ) depends on the particular familiarization stimuli and on how those stimuli are presented (French, Mermillow, Quinn, \& Mareschal, 2001; Oakes, Coppage, \& Dingel, 1997; Quinn et al., 1993). Thus, infants' responding in familiarization does not reflect an underlying concept but reflects similarities noticed in the context of the task. Forming categorical groups in this context, therefore, seems to involve stimulus discrimination and generalization.

Research investigating infants' categorization of instances from artificial categories created by the experimenter (e.g., line drawings of made-up animals) does show that infants form categorical representations on line in the context of an experimental session; because the exemplars and categories are constructed by the experimenter, infants cannot have formed the categorical representation before entering the lab. Infants familiarized with a set of unfamiliar objects from a novel category can extract prototypes of those stimuli (e.g., Strauss, 1979) and can use correlations among attributes to categorize those stimuli (e.g., Younger \& Cohen, 1986). However, the ways in which these artificially constructed stimuli are similar may not correspond to ways in which items from everyday categories (e.g., dog or cup) are similar. Thus, it is not clear how generalizable these results are to infants' categorization of more realistic stimuli.

The goal of the present investigation was to show that infants form novel categorical representations in the lab even when presented with objects for which they may have previously formed categorical representations. We induced infants to form a new categorical representation of realistic animal stimuli (black-and-white animals) during an experimental task. This category does not reflect an enduring, stable representation: Although some animals are black and white in coloring (e.g., zebras or cows), animals are not typically grouped in this way (this perceptual similarity tells us nothing about where the animals live, how they behave, or what they eat), and it is, therefore, unlikely that infants (or adults) have previously formed a categorical representation that includes only black-and-white animals. Demonstrating that infants can form such categorical representations provides a strong test of the hypothesis that infants, like adults, can rapidly form categorical representations on line in the context of familiarization and shows that there are similarities in at least some of the processes of categorization across the lifespan.

We familiarized infants with realistic three-dimensional replicas of animals, and thus, the items varied in the ways in which real objects from natural categories vary. Importantly, we did not assume that infants' categorization of these stimuli reflects their use of an animal concept. Rather, we used realistic stimuli, because they mimic the kind of variability found among exemplars in real-world categories (as opposed to laboratory categories), and thus, we have confidence that the processes uncovered can be used in infants' everyday interactions with objects.
We manipulated one aspect of similarity among the items: the overall coloring. We familiarized 6-, 10-, and 13-month-old infants with a set of land animals that were all black and white in coloring and then tested their responses to a novel black-and-white land animal, a novel land animal of different coloring, a sea animal, and a truck. For comparison, we familiarized a separate group of infants with a set of land animals that were more variable in coloring and then tested their responding to the same tests. Because this set of animals was not characterized by common coloring, infants should form a more inclusive categorical representation - for example, a category that includes animals of any color. Note, therefore, that both groups of infants were familiarized with essentially the same set of animals, but the experimental context was manipulated to bias infants in the black-and-white condition to respond to a new category that included only the novel black-and-white animal and infants in the variable condition to respond to a broader category that included many different types of novel animals.

\section{METHOD}

We tested healthy full-term 6-month-old (12 boys and 12 girls; mean age, 29.65 weeks; $S D=0.85), 10$-month-old $(11$ boys and 13 girls; mean age, 44.10 weeks; $S D=1.12$ ), and 13-month-old ( 12 boys and 12 girls; mean age, 59.56 weeks; $S D=1.24$ ) infants. Twenty-nine additional infants were tested but were excluded from the final sample because of fussiness $(n=7)$, videotaping equipment malfunction or experimenter error (failure to videotape the session, presenting the wrong number of familiarization trials or the wrong test items; $n=18)$, or parental interference $(n=4)$. The infants were recruited using standard procedures (e.g., Oakes et al., 1997).

The infants were seated on a parent's lap at a table directly across from an experimenter. On each of 1630 -sec trials, the experimenter presented a single toy to the infant and said, "Can you play with this?" The infants were allowed to manipulate the toy in any way they desired. If the toy fell off the table, it was quickly returned to the infant (trial durations were not adjusted). The first 12 trials were familiarization trials, during which the infants received trials with four different hard plastic land animals (each approximately 12$15 \mathrm{~cm}$ in length). Each object was presented once in each block of four randomly ordered familiarization trials. Half of the infants at each age were familiarized with four exemplars drawn from a set of five black-and-white land animals (cow, dog, panda bear, zebra, and tiger), and the other infants were familiarized with four exemplars drawn from a set of five land animals that varied in coloring (brownand-white cow, brown, black, and white German shepherd, brown bear, light yellow horse, and yellow, brown, and orange tiger; see Figure 1). Note that each animal had a match in the other set (e.g., a panda bear and a brown bear).

The last four trials were test trials. First, the infants received tests with a novel black-and-white land animal and a corresponding land animal from the variable set (e.g., zebra and horse), presented individually in an order that was counterbalanced across infants. The infants' responding to these tests would assess their inclusion of blackand-white and more varied land animals into their categorical representation. Previous research has shown that infants look longer at members of a new category, and this was our measure of category inclusion or exclusion. Specifically, if infants familiarized with black-and-white animals form an exclusive categorical representation, they should include only the black-and-white land animal and look longer at the novel variable animal. Infants familiarized with a more variable set of animals are expected to form a broader cate- 


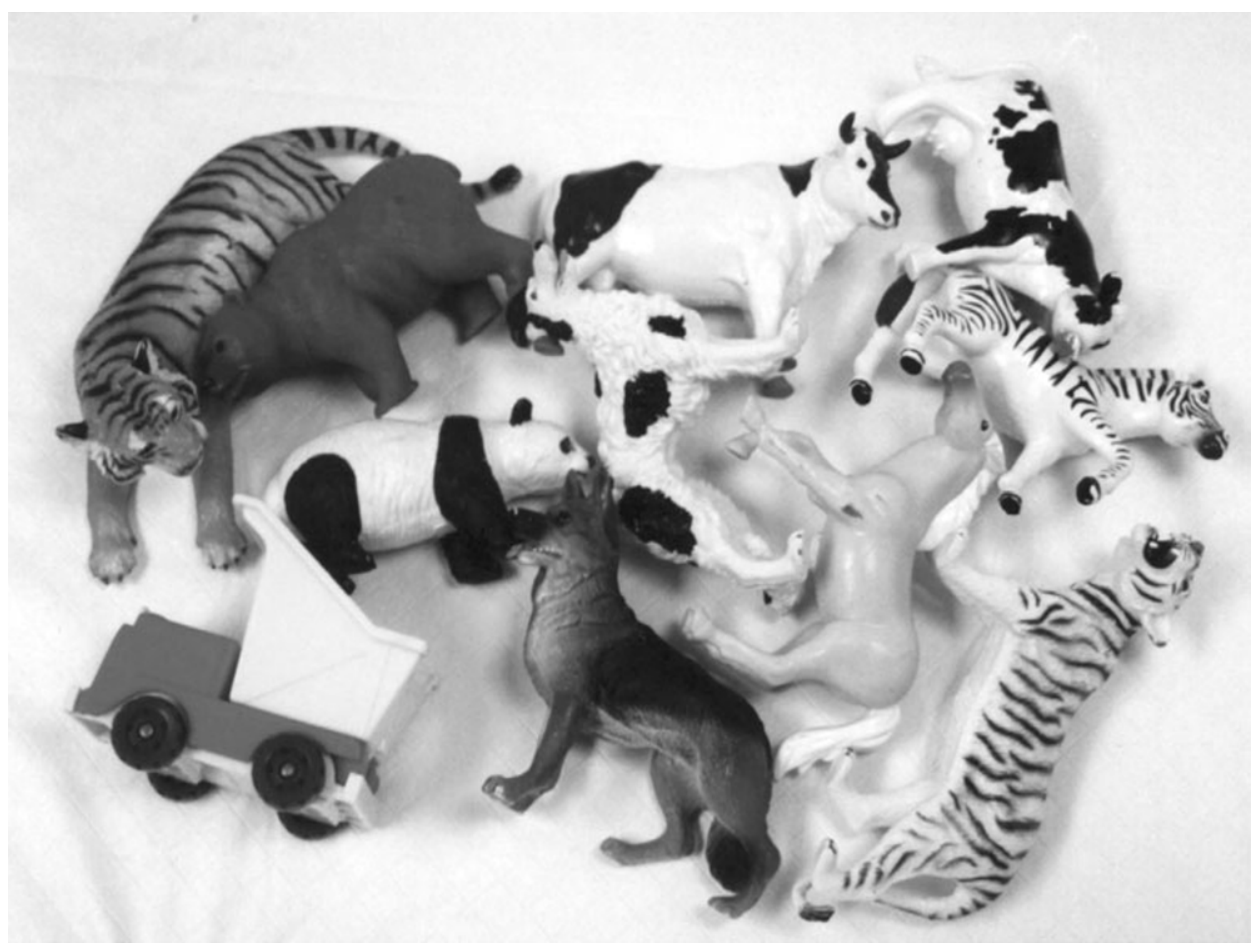

Figure 1. The plastic replicas of land animals used as stimulus objects. Half of the animals were the blackand-white set, and half were the variable set. Note that each black-and-white item has a corresponding item in the variable set (e.g., there is a black-and-white dog and a brown-and-black German shepherd).

gorical representation, and thus, they will look equally long at both novel land animals.

We further tested the boundaries of the categorical representations formed in this experiment by assessing the infants' responses to a novel sea animal (approximately $22 \mathrm{~cm}$ in length) on the third test trial (37 infants received a blue dolphin; 35 infants received a black, gray, and white humpback whale) and a truck (approximately $9 \mathrm{~cm}$ in length) on the fourth test trial. If infants in either condition form a broad category, they should respond in the same way to the two novel land animals and the sea animal, but they should look longer at the truck. This is the pattern we expected for the infants familiarized with the varied set of land animals.

The videotaped sessions were edited to allow separate coding of familiarization and test. Therefore, for the test trials, coders recording the duration of looking did not know whether the infant had been familiarized with the black-and-white or the variable items. Naive coders viewed videotapes of the sessions and pressed and held a computer key as long as an infant was clearly looking at the item presented on each trial. Reliability was assessed for approximately $25 \%$ of each sample; correlations for the duration of looking on each trial were high $(\geq .97)$ and the average difference in looking (on 30sec trials) was low $(0.70-1.28 \mathrm{sec})$.

Before analyzing the data from the main experiment, we conducted two important controls. First, we established that infants could actually discriminate the items within each familiarization set by testing a separate group of 23 6-month-old and 24 10-month-old infants, using the procedure described above, except that the infants were familiarized with a single exemplar drawn either from the black-andwhite or the variable set of land animals. This control revealed that, indeed, infants could discriminate the items in both familiarization

Table 1

Means (in Seconds) and Standard Deviations for Infants in the Discrimination Control Task (Difference Scores) and Preference Control Task (Looking Times)

\begin{tabular}{|c|c|c|c|c|c|c|c|c|c|c|}
\hline \multirow[b]{2}{*}{ Control Task } & \multirow[b]{2}{*}{ Group } & \multirow[b]{2}{*}{$N$} & \multicolumn{2}{|c|}{$\begin{array}{l}\text { Black-and-White } \\
\text { Land Animal }\end{array}$} & \multicolumn{2}{|c|}{$\begin{array}{c}\text { Variable } \\
\text { Land Animal }\end{array}$} & \multicolumn{2}{|c|}{ Sea Animal } & \multicolumn{2}{|c|}{ Truck } \\
\hline & & & $M$ & $S D$ & $M$ & $S D$ & $M$ & $S D$ & $M$ & $S D$ \\
\hline \multirow[t]{2}{*}{ Discrimination } & Familiarized to black-and- white land animal & 23 & $5.41 * *$ & 8.54 & $8.62 * *$ & 7.08 & $6.54 * *$ & 7.54 & $14.93^{* *}$ & 5.88 \\
\hline & Familiarized to variable land animal & 24 & $8.40^{* *}$ & 7.70 & $6.66^{* *}$ & 7.35 & $4.63^{*}$ & 8.44 & $13.32 * *$ & 6.69 \\
\hline \multirow[t]{2}{*}{ Preference } & Black-and-white land animal & 30 & $15.89^{\mathrm{a}}$ & 5.56 & & & 15.10 & 6.14 & $18.44^{\mathrm{b}}$ & 5.71 \\
\hline & Variable land animal & 30 & & & 15.96 & 6.24 & 15.34 & 5.72 & 17.45 & 5.46 \\
\hline
\end{tabular}

Note-For the discrimination control task, to determine whether infants significantly increased their looking to the test relative to their looking at the end of familiarization, difference scores (looking to the test minus looking during the last familiarization trial) for each test were compared with zero. Scores that differed significantly from zero are indicated by a single asterisk $(p<.05)$ or a double asterisk $(p<.01)$, two-tailed. For the preference control task, to determine whether infants significantly preferred one item over another, within-group comparisons were conducted on the looking times for the three items. Within each group mean, looking times that differed significantly $(p<.05)$ are indicated by different superscripts. 
sets. The analysis of the infants' looking times during each block of four familiarization trials revealed a significant main effect of trial block $[F(2,86)=28.01, p<.01]$, due to the infants' decreasing their looking during familiarization, and a main effect of age $[F(1,86)=$ $5.30, p<.05]$, due to 6 -month-old infants looking longer overall during familiarization than did 10-month-old infants, a general trend reported in other studies (e.g., Oakes \& Tellinghuisen, 1994). The infants increased their looking time to each of the tests. Difference scores were calculated by subtracting the duration of looking during the last familiarization trial from the duration of looking during each test (see Table 1). Comparison of these scores with zero showed that the infants in both conditions increased their looking time significantly to all the novel items.

Next, we evaluated infants' a priori preferences for the test items. We presented an additional 60 infants (20 each at 6,10 , and 13 months) with three 30 -sec trials - one with a sea animal, one with a land animal, and one with a truck (order completely counterbalanced across infants). For half of the infants, the land animal was drawn from the black-and-white set, and for the other infants, the land animal was drawn from the variable set. The infants' mean looking time for each item is presented in Table 1 . There were no systematic a priori preferences. The infants looked approximately equally to the black-andwhite land animal and the sea animal and approximately equally to the variable land animal, the sea animal, and the truck. The infants did look significantly longer at the truck than at the black-and-white animal, but the magnitude of this difference was considerably smaller than that exhibited by the infants in the main experiment (see below). In general, therefore, any differences in looking in the main experiment are the result of the familiarization procedure and not the result of systematic a priori preferences for any of the items.

\section{RESULTS}

An analysis of variance (ANOVA) conducted on the average duration of looking in each block of four familiarization trials, with age and familiarization condition as between-subjects factors, revealed only a main effect of block $[F(2,132)=39.90, p<.01]$. The infants significantly decreased their looking time from Block $1(M=17.54$, $S D=5.13)$ to Blocks $2(M=14.79, S D=4.96)$, and 3 ( $M=13.04, S D=4.85 ; p<.05$, using Tukey's HSD). Because the ANOVA revealed no main effects or interactions involving condition or age, there was no indication that the infants' attention to the toys varied as a function of age or the particular set of familiarization items. It may seem surprising that the increased within-category similarity in the black-and-white condition did not lead to faster learning of the category and, thus, a faster rate of decrease during familiarization. However, we assume that the infants formed a categorical representation in both conditions, and the lack of an interaction may reflect this similarity in process.

Next, we evaluated the exclusivity of the categorical representation formed in each condition by evaluating the degree of increase in looking time to each of the tests. Difference scores (looking during test - looking during last familiarization) for each test show that the infants familiarized with black-and-white animals increased their looking time to all the novel objects, except for the novel black-and-white land animal, and the infants familiarized with the more variable set of land animals increased their looking time somewhat to the sea animal and clearly to the truck (see Figure 2). The infants in the black-andwhite familiarization condition apparently formed a nar- row categorical representation, and the infants in the variable familiarization condition apparently formed a broader categorical representation. This conclusion was confirmed by an ANOVA conducted on the difference scores, with age and condition as between-subjects factors and test trial (novel black-and-white, novel variable, and sea animals) as a within-subjects factor. This analysis revealed only a significant test trial $\times$ condition interaction $[F(2,132)=$ $3.56, p<.05]$, confirming that the infants in the two conditions exhibited different patterns of responding to the tests. (Note that no developmental differences were observed, but rather, the same general pattern was observed at all three ages. The infants' ability to rapidly form a novel categorical representation of these stimuli in this task appears to undergo little developmental change between 6 and 13 months.)

We explored this interaction by conducting separate one-way ANOVAs on the difference scores for each condition. The ANOVA from the black-and-white condition revealed a main effect of trial $[F(2,66)=5.98, p<.01]$, and Tukey's HSD comparisons of the means revealed that the difference scores for the novel variable item and the sea animal were greater than the difference score for the novel black-and-white animal $(p<.05)$. The ANOVA from the variable condition revealed no significant effects, indicating that the infants responded similarly to all three tests. Therefore, the infants familiarized with black-andwhite animals responded differently to the novel animals (apparently including only the novel black-and-white land animal in their categorical representation), and the infants familiarized with more variable animals responded equivalently to all the novel animals.

We also compared the difference scores for each test stimulus with zero. If infants exclude a particular item from a categorical representation formed during familiarization, their increase in looking time for that item should be significantly greater than zero. These comparisons revealed that the infants in the two groups excluded different items from the categorical representation they formed. The infants in the black-and-white condition significantly increased their looking time for the novel variable exemplar $[t(35)=3.38, p=.002]$, the sea animal $[t(35)=$ $3.37, p=.002]$, and the truck $[M=10.51, S D=7.65$; $t(35)=8.25, p<.001]$, but not for the novel black-andwhite exemplar $[t(35)=1.04, p=.31]$. (The infants' increase in looking time for the blue dolphin was not different from their increase for the black, gray, and white whale.) The infants in the variable condition, in contrast, did not increase their looking time significantly for the novel black-and-white exemplar $[t(35)=1.27, p=.21]$, the novel variable exemplar $[t(35)=0.67, p=.50]$, or the sea animal $[t(35)=1.95, p=.06]$, but they did increase their looking time significantly for the truck $[M=9.85$, $S D=7.87 ; t(35)=7.51, p<.001]$. Thus, these infants apparently included all of the animals used here in their categorical representations. (Note that they did increase their looking time somewhat to the sea animal. However, regardless of whether these infants included or excluded sea animals, they clearly formed a more inclusive categor- 


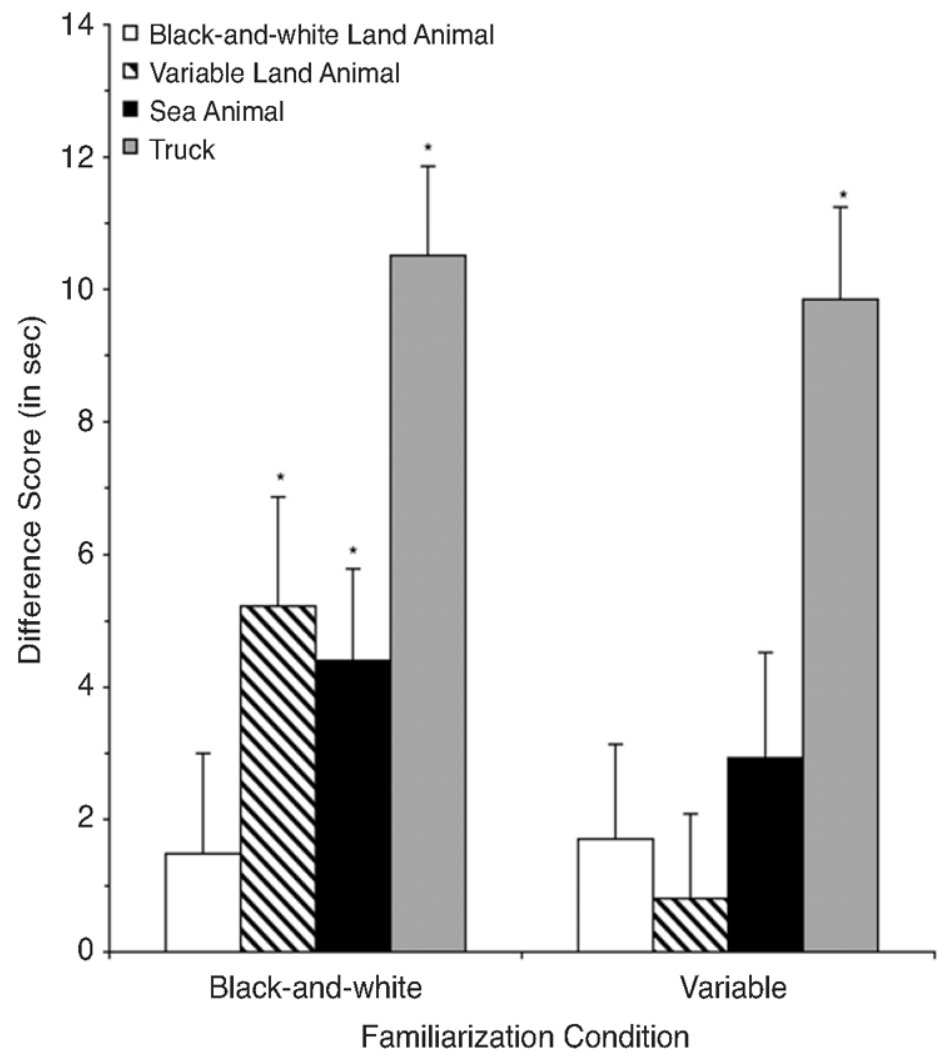

Figure 2. Increase in looking time, as represented by difference scores (duration of looking during test - duration of looking during last familiarization trial), for the animal tests by condition (error bars $=+1 S E$ ). Increases that are significantly greater than zero are indicated by an asterisk.

ical representation than did the infants familiarized with black-and-white animals.) Thus, the infants in the blackand-white condition formed a very exclusive categorical representation; they treated as novel any exemplar that was not a black-and-white land animal. The infants in the variable condition, in contrast, formed an inclusive category.

\section{DISCUSSION}

The present results show that infants can rapidly form new categorical representations of realistic stimuli. Infants' responding during test, therefore, does not solely reflect stable, underlying categorical representations. Rather, infants form categorical representations on line during familiarization, depending on the kinds of similarities among the exemplars presented. This conclusion is similar to that drawn by Smith and her colleagues (Jones \& Smith, 1993; Smith, 2000). In particular, Smith has argued that categorization, as assessed in habituation or familiarization, taps the process of forming categorical representations, rather than tapping existing categorical representations that infants have before coming into the lab. The present data confirm this conclusion: the infants' categorical representations were task dependent. They formed a different categorical representation when familiarized with black-and-white land animals than they did when familiarized with a more variable set of land animals.

Importantly, this study represents a shift in the kinds of questions we ask about infants' categorization. Rather than asking whether infants respond to natural adult-defined categories (which may or may not be preexisting), we asked whether infants form new categorical representations in response to the experimental context. This emphasis is different from that of investigators such as Mandler (2000), who has suggested that the task used here taps infants' existing conceptual categories of objects. In this case, the infants in both conditions should have responded to the same category, because all the infants were familiarized with land animals. In accord with the results of previous studies (French et al., 2001; Oakes et al., 1997; Quinn et al., 1993), however, the present results show that infants form different categorical representations depending on the particular familiarization stimuli, even when those stimuli are drawn from the same adult-defined category. Infants used different perceptual similarities to form a categorical representation when familiarized with black-and-white land animals than when familiarized with variable land animals. These results provide a particularly strong test of the hypothesis that infants' categorization, like adults', is context dependent. 
Obviously, because we can present infants only with a small number of test items, we cannot determine from the present results the exact boundaries of the categorical representations formed in the two conditions. The infants' responding to the tests may have reflected their recognizing the similarity among the animals in terms of color, size, shape, or some combination of these features. However, recognition of such similarities is central to categorization, and therefore, the results clearly show that infants' responding in this task need not reflect a deep conceptual understanding of the familiarization items. Moreover, because the infants in the two conditions responded differently to the test items, we can conclude with confidence that familiarization with a set of black-and-white land animals induced the infants to form a categorical representation different from that formed from familiarization with a more variable set of land animals.

These results have implications for how we interpret previously reported results of infants' categorization in familiarization tasks. Specifically, because the infants' responding to test items appears to have reflected contextdependent categorical representations, it is possible that infants' responding in previous studies also reflected representations formed on line during the experiments, rather than solely reflecting preexisting representations. That is, the infants' responding in this experiment clearly did not reflect their conceptual representations of the adultdefined categories of animals or land animals; rather, infants' responding to such categorical groupings in tasks such as those employed here reflects (in part) their on-line formation of task-specific categorical groupings based on perceived similarities among items. Infants' previous experience with animals may influence the kinds of categorical representations they form. However, the present findings show that infants' responding in this task reveals task-specific categorical representations. We must be cautious, therefore, in drawing conclusions about what categories infants possess on the basis of their responding in familiarization tasks.

Finally, it is important to ask what these results tell us about the development of categorization in infancy. Because no developmental differences were observed, these results may at first appear to provide little developmental insight. However, the present results show that infants, like adults, are able to rapidly create task-specific categorical representations, even when presented with realistic items. Thus, consistent with a general theoretical framework in which the basic processes of categorization do not change but, rather, there are changes in the kinds of information infants use in categorizing (Oakes \& Madole,
2000), the present results reveal continuity in categorization across the lifespan.

\section{REFERENCES}

French, R. M., Mermillow, M., Quinn, P. C., \& Mareschal, D. (2001). Reversing category exclusivities in infant perceptual categorization: Simulations and data. In K. Stenning \& J. Moore (Eds.), Proceedings of the 23rd Annual Conference of the Cognitive Science Society (pp. 307-312). Mahwah, NJ: Erlbaum.

GoLDSTONE, R. L. (1995). Effects of categorization on color perception. Psychological Science, 6, 298-304.

Jones, S. S., \& SMITH, L. B. (1993). The place of perception in children's concepts. Cognitive Development, 8, 113-139.

MANDLER, J. M. (2000). Perceptual and conceptual processes in infancy. Journal of Cognition \& Development, 1, 3-36.

MANDler, J. M., \& McDonough, L. (1993). Concept formation in infancy. Cognitive Development, 8, 281-318.

OAKes, L. M., Coppage, D. J., \& Dingel, A. (1997). By land or by sea: The role of perceptual similarity in infants' categorization of animals. Developmental Psychology, 33, 396-407.

OAKES, L. M., \& MADOLE, K. L. (2000). The future of infant categorization research: A process-oriented approach. Child Development, 71, 119-126.

OAKES, L. M., \& Tellinghuisen, D. J. (1994). Examining in infancy: Does it reflect active processing? Developmental Psychology, $\underline{\mathbf{3 0}}_{2}$ 748-756.

QUinN, P. C., \& Eimas, P. D. (1997). A reexamination of the perceptualto-conceptual shift in mental representations. Review of General Psychology, 1, 171-187.

Quinn, P. C., Eimas, P. D., \& Rosenkranz, S. L. (1993). Evidence for representations of perceptually similar categories by 3-month-old and 4-month-old infants. Perception, 22, 463-475.

Ross, G. S. (1980). Categorization in 1- to 2-year-olds. Developmental Psychology, 16, 391-396.

Roth, E. M., \& SHOBEn, E. J. (1983). The effect of context on the structure of categories. Cognitive Psychology, 15, 346-378.

SMith, L. B. (2000). From knowledge to knowing: Real progress in the study of infant categorization. Infancy, 1, 91-97.

Spalding, T. L., \& Murphy, G. L. (1996). Effects of background knowledge on category construction. Journal of Experimental Psychology: Learning, Memory, \& Cognition, 22, 525-538.

STRAuSs, M. S. (1979). Abstraction of prototypical information by adults and 10-month-old infants. Journal of Experimental Psychology: Human Learning \& Memory, 5, 618-632.

Younger, B. A., \& COHEN, L. B. (1986). Developmental changes in infants' perception of correlations among attributes. Child Development, $\underline{\mathbf{5 7}}, \mathbf{8 0 3 - 8 1 5}$.

\section{NOTE}

1. We refer to the categories of animals and land animals in this article as a shorthand to describe the particular groups of items to which the infants were familiarized. The use of these terms should not be taken to indicate that infants possess or create representations equivalent to the adult-defined categories.

(Manuscript received August 30, 2002; revision accepted for publication July 21, 2003.) 